\title{
DELONE SETS AND MATERIAL SCIENCE: A PROGRAM
}

\author{
JEAN BELLISSARD
}

\begin{abstract}
These notes are proposing a program liable to provide physicists working in material science, especially metallic liquids and glasses, the mathematical tools they need to build an atomic scale theory of Continuous Mechanics including plasticity, fluidity and, hopefully, fractures. Using the long list of datas and numerical simulations accumulated during the last forty years, physicists have identified a new class of degrees of freedom, besides the elastic ones, which will be called anankeons here [7]. They are dominant in the liquid phase and they explain the properties related to plastic deformations of the solid phase. It is advocated that Delone sets provide a natural frame within which such a theory can be expressed. The use of Voronoi tiling and its dual construction, called the Delaunay triangulation, gives a discretization of the data. The concept of Pachner move or Delaunay flips permits to describe very precisely what the anankeons are. A partition of the configuration space into contiguity domains leads to a graph on which a Markov process can be built to describe the anakeon dynamics. At last, a speculative Section is giving an attempt to describe the Continuous Mechanics of a condensed material in terms of a Noncommutative Geometry of the configuration space.
\end{abstract}

\section{INTRODUCTION}

This article is an attempt to explain how the mathematical tools used in the study of tiling spaces can be used to describe very concrete materials like liquids and glasses. The text will then have a part describing the situation viewed from the eye of the physicists or the material scientist, and another part consisting in describing the mathematical material. As a consequence, the reader who is not an expert in Physics or in Statistical Mechanics, may need some time to adjust and understand the content. The author apologizes for demanding so much from the reader. However, developing a mathematical tool liable to describe the real world is never an easy task because it always includes several important aspects requiring to use pieces of Mathematics that have usually little to do with each other.

1.1. A Program. Describing a material in a condensed phase, solid or liquid, from the atomic scale on is still a challenge today. These notes provide a guideline liable to fill this gap in the future. The main new ingredient is the concept of anankeon $[1,7]$. This is the dominant degree of freedom for liquids, while anankeons play a crucial role in solids to account for their plasticity. As will be seen, this concept of anankeon fits with a description of atomic configurations in terms of Delone sets, Voronoi tiling, Delaunay triangulation. Anankeon then appear to be any of the individual simplex of this triangulation. Changes in the local triangulations, known as Pachner moves or Delaunay flips, give a way to describe the anankeon dynamics. However the latter is essentially unpredictable and has to be given through a Markov process. Numerical simulations,

Work supported in part by NSF grant DMS 1160962.

The author is especially indebted with T. Egami for many exchanges of idea since February 2012. He is also indebted with J. Langer who accepted to have a long discussion in May 2013 about his ideas on the STZ theory. 
based on molecular dynamics are suggesting various phenomenological forms for this Markov process.

In the last Section of these notes a list of speculations will be presented in form of a program. The author believes, indeed, that the mechanical properties of a condensed material should have a geometrical interpretation. He will argue that the Geometry is not in the usual space but in the Transversal direction of the Hull of the set of atomic configurations. The argument will be given along the following lines. The generator of a Markov process is usually considered as a Dirichlet form acting on a Hilbert space $[51,52,59]$. In the present situation, however, it is expected to be rather the generator of a Markov semigroup on an abelian $C^{*}$-algebra $\mathcal{A}$ attached to Transversal. The invariance of $\mathcal{A}$ by the Markov semigroup is the analog of the Feller property in the theory of Markov Processes. It will be argued that, in such a case, it should be possible to define a metric on the tiling space in a way similar to the constructions of Kantorovich [61, 62] or Connes [55]. It is then expected to provide the tiling space with a Noncommutative analog of a Riemannian manifold. The main issue discussed at the end is the definition of the curvature. This concept is still ill-defined in Noncommutative Geometry. However, the curvature is expected to be related to the mechanical properties of the material, through some kind of Einstein equation:

\section{Curvature $=$ Stress}

1.2. Some Physics Background. The usual approach to solids and liquids is to distinguished between scales: length scales, time scale, energy scales. At macroscopic length scales either the Elasticity Theory for solids (see for instance [14, 8]), or the Navier-Stokes equation for fluids, give the standard description. At a lower scale, a kinetic theory approach, using the Bolztmann equation or the BBGKY hierarchy, is also considered (see for instance [11]). While kinetic theories have been successful for gas, they are more difficult to manipulate for liquids. Since the late nineties two major theoretical contributions were made to improve the description of solids at large scale. The first is due to a group of physicists led by J. Langer, and is called the STZ Theory, where STZ stands for shear-transformation zones $[9,10,15,16,17]$. It proved to be very efficient in describing the plasticity region in the strain-stress curve, to describe also the evolution of fractures $[20,19]$ in glassy systems. The other one, more phenomenological, is called Peridynamics Theory and was initiated by Silling $[21,22,23]$. It became recently important in particular to describe solid under extreme stress, like explosives.

The discovery of a new class of materials, during the nineties, called bulk metallic glasses (BMG), like the vitalloys containing mostly copper and zirconium, has been an experimental and a technological breakthrough $[12,13]$ : these materials have exceptional mechanical properties, a very high limit of elasticity and an exceptional resistance to rupture. They are now used in various devices including sport material (i-Phone6, golf clubs, ... ) and medical tools. The development of these materials has triggered more research, has attracted funding and therefore it provides a window of opportunity for theoreticians and experimentalists alike to test ideas and models liable to give access to a better understanding of both glass and liquid phases.

1.3. Lessons from Available Datas. Several experts in the field of BMG have expressed the wish to develop a more universal modeling valid from the atomic scale on with the help of mathematicians. There are several layers of difficulties in this problem. In the fifties, Bernal used a Voronoi construction to describe the atomic configurations of such materials [3]. In the early eighties, the local cluster theory provided a more detailed description of the local environment of a typical atom. This was initiated by T. Egami et al. [6] and was completed by Miracle [18] 
during the last decade. The main experimental tool to analyze the results of this approach is the diffraction spectrum (X-ray, electrons or neutrons), the Fourier transform of which permits to compute the pair distribution function (PDF).

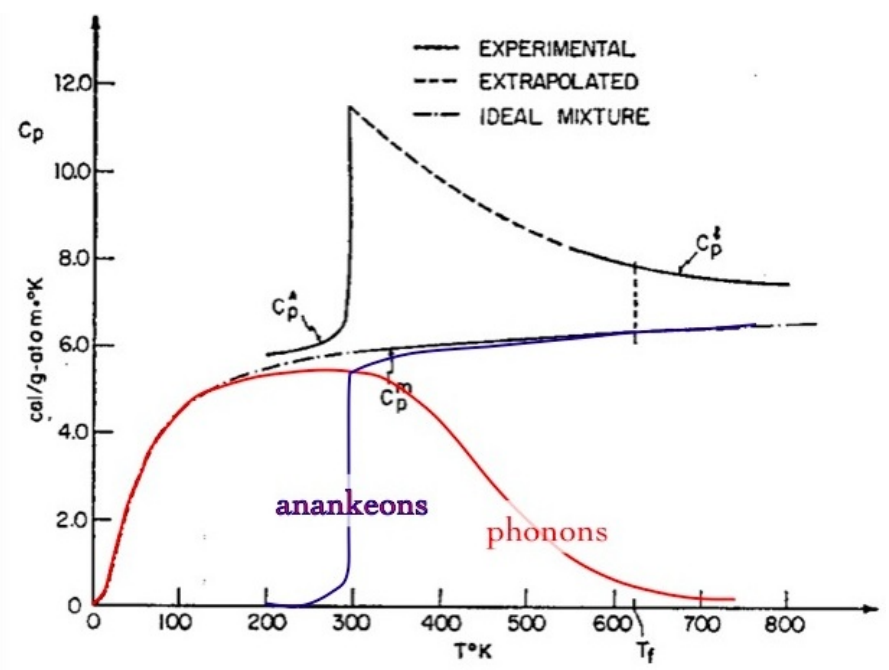

Figure 1. Heat capacity as a function of temperature for an alloy made of Gold, Germanium and Silicon signaling a glass-liquid transition [4]. In color a possible contribution of phonons and anankeons

A liquid phase approach has also been advocated for a long time, in order to identify the most relevant degrees of freedom. The heat capacity is an observable easy to measure in experiments. It provides some informations about how the energy is stored within various degrees of freedom. The behavior of the heat capacity of a typical disordered material (see Fig. 1) reveals a low temperature regime in the solid phase (glass), a saturation at high temperature (liquid phase) leading to a similarity with the law of Dulong-Petit and a transition region (liquid-glass transition) showing an increase of the heat capacity which looks like a phase transition without a singularity. The Law of Dulong-Petit predicts that the heat capacity per unit volume of a solid saturates at high temperature and is given by

$$
C_{V}=M k_{B}, \quad M=\text { number of degrees of freedom, }
$$

and $k_{B}$ is the Boltzmann constant. Einstein assumed that the main degrees of freedom in a solid where provided by phonons, namely the quantized version of acoustic waves, leading to $M=3$. However, it is known that phonons are mainly damped in liquids so that the experimental result showed in Fig. 1 requires an explanation. The anakeon theory might give an interpretation of these observations (see Section 3.1 below).

The second layer of difficulty is the time dependence of the response to mechanical strain. For example diving into water by jumping from the edge of a swimming pool is possible because the water moves fast enough to permit the diver's body to enter smoothly. However, firing a bullet in water results in the bullet being completely smashed like if water was a piece of concrete. In the former case, the speed of the diver is low, permitting the water to react fast enough, while in the latter case, the speed of the bullet is so high that the water is too slow to adjust around the bullet and behave like a very hard solid. In more scientific terms, the response to shear involves a time scale that is usually expressed through the viscosity of the medium. In particular, the 
main difference between liquid and glass is not seen in the equilibrium parameters like density or heat capacity. It is mainly seen in a sudden increase of the viscosity. Namely the time scale characterizing the response to shear increases by several orders of magnitude within a very small interval of temperature at the transition. The challenge in this case is to provide a theory liable to describe the dynamics of the atomic configurations.

1.4. Degrees of Freedom. Over the years, the work of Egami and his collaborators has permit to identify two relevant classes of degrees of freedom at the atomic scale.

(1) the vibration degrees of freedom, the one at work in the theory of elasticity, at least for long wave length or the ones with short wave-lengths that can eventually be quantized and which has been called phonons by A. Einstein in 1908. In the glass phase, phonon seem to give the dominant contribution. However in the liquid phase the high frequency phonons are damped, with a very short lifetime.

(2) the topological degrees of freedom, representing the unpredictable sudden change in the local configuration of atoms due to the local stress and the thermal motion. These degrees of freedom are newcomers in the theoretical landscape and will be called anankeon here [1]. They provide an explanation to the Dulong-Petit behavior of the heat capacity [5] (see Fig. 1), namely the saturation at large temperature. They just freeze at the liquidglass transition. They reappear only if an external shear is applied as the atomic scale version of the STZ proposed by Langer et al..

While the dynamics of phonons is well understood, including nonlinearity if necessary, the dynamics of anankeon is not. A model for this dynamics, using a Markov process, will be proposed. The construction of such a process uses the Boltzmann-Gibbs factor for the equilibrium dynamics as well as the Arrhenius law for the evaluation of waiting times. Recent numerical simulations made by T. Egami and his group of collaborators, give a hint about the actual magnitude of the various parameters, energy, length and time scales involved in such a modeling.
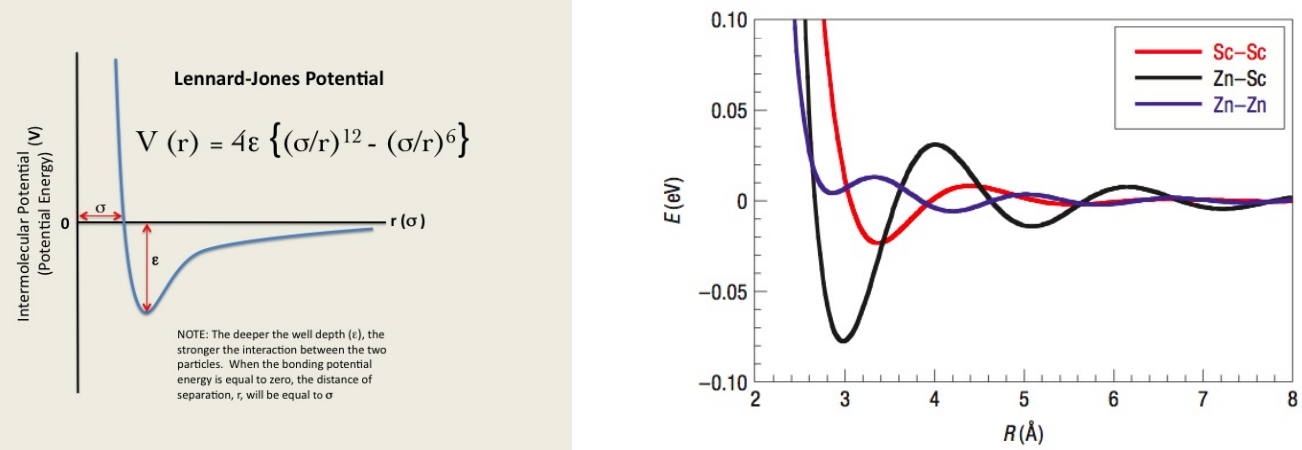

FiguRE 2. Left: a representation of the Lennard-Jones potential; Right: an effective interatomic potential computed numerically for an $Z n-S c$ alloy.

\section{Why Delone Sets ?}

2.1. Interatomic potential. Even though atoms, especially in transition metals like copper or zirconium, have a complicated structure with many electrons around the nucleus, it is a convenient and usual approximation to consider them as individual particles interacting through 
a pair-potential. This potential is usually a function of the relative position of the atoms having the following properties: (i) it is repulsive at short distance and (ii) attractive at large distance. In addition, for most isotropic materials, it can be assumed that this potential is rotation invariant, meaning that it is a function of the interatomic distance $r$. Examples used in numerical simulations, using molecular dynamic, for instance, are the Lennard-Jones potential, valid mostly for systems interacting with the van der Waals force, such as rare gases, the Johnson one which is short range and exhibit oscillations or potential computed ab initio for metallic alloys (see Fig. 2). Apparently the results of simulations are not strongly affected by the nature of the potential. The origin of such potential, from first principle will not be discussed here. However they provide some concrete length scales: (i) a short length scale $\sigma$ such that for $r<\sigma$ then $V(r)>0$, (ii) the minimum potential length $a$. It is expected that atomic configurations with two atoms closer than $\sigma$ are rare. In addition, atomic configurations with a vacancy bigger than $a$ are unstable w.r.t. shear (see Fig. 4 and a discussion in [25]), giving a limit for the typical size of a vacancy.

2.2. Quasi-discrete Measures. A configuration of atoms can be represented by the positions of its atomic nuclei. Namely, if $\mathcal{A}$ denotes the set of atomic species present in the material, the position of atoms of the species $a$ make up a discrete sets of points $\mathcal{L}_{a} \subset \mathbb{R}^{d}$ where $a \in \mathcal{A}$. Such a set is topologically closed, meaning that it has no accumulation point. It is mathematically better represented by the corresponding sum of Dirac measures $\delta_{x}$ located at the position of the atomic nuclei, namely by $\nu_{a}=\sum_{x \in \mathcal{L}_{a}} \delta_{x}$. Such a measure are called discrete and can be characterized by the following properties [2]:

(i) it is a Radon measure on $\mathbb{R}^{d}$,

(ii) given any ball $B \subset \mathbb{R}^{d}$ the measure $\nu_{a}(B)$ of this ball is an integer for each $a \in \mathcal{A}$.

(iii) the measure of a point $\nu_{a}\{x\}$ is either 0 or 1 .

What is a Radon measure ? It is a way to evaluate weighted integral of functions. Which functions ? Any complex valued function $f$ defined on the physical space $\mathbb{R}^{d}$ (i) which is continuous and (ii) which vanishes outside of some bounded ball (namely $F$ has compact support). The space of such functions is denoted by $\mathcal{C}_{c}\left(\mathbb{R}^{d}\right)$. Then the integral

$$
\mu(f)=\int_{\mathbb{R}^{d}} f(x) \mu(d x)
$$

has the following properties: (i) $\mu(f)$ is linear in $f$, (ii) if $\left(f_{n}\right)_{n=1}^{\infty}$ is a sequence of function in $\mathcal{C}_{c}\left(\mathbb{R}^{d}\right)$ which are all supported a common ball $B$ and converging uniformly on $B$ to a function $f$ (that is to say $\lim _{n \rightarrow \infty} \sup _{x \in B}\left|f(x)-f_{n}(x)\right|=0$ ), then $\mu(f)=\lim _{n \rightarrow \infty} \mu\left(f_{n}\right)$. In the previous case

$$
\nu_{a}(f)=\int_{\mathbb{R}^{d}} f(x) \nu_{a}(d x) \stackrel{\text { def }}{=} \sum_{x \in \mathcal{L}_{a}} f(x) .
$$

The set of Radon measures is equipped with its weak ${ }^{*}$-topology, meaning that a sequence $\left(\nu_{n}\right)_{n \in \mathbb{N}}$ of such measures converges to a measure $\nu$ if and only if $\nu_{n}(f) \rightarrow \nu(f)$ for any complex valued continuous function $f$ on $\mathbb{R}^{d}$ with compact support. As it turns out, the set of discrete measures described above is not weak ${ }^{*}$-closed. Its closure is made of measures of the form $\nu=\sum_{x \in \mathcal{L}} n_{x} \delta_{x}$, where $\mathcal{L} \subset \mathbb{R}^{d}$ is closed and discrete, while the $n_{x}$ 's are integers. Namely it describes configurations of atoms in which a finite number of atoms are allowed to occupy the same position. This weak*-closure is denoted by $\mathrm{QD}_{\mathcal{A}}\left(\mathbb{R}^{d}\right)$, where $\mathrm{QD}$ stands for quasi-discrete. It is a Polish space 
[2]: namely, its topology can be described through a metric for which it is complete, while such a metric is neither unique nor canonical. Polish spaces allow for a good theory of probability [36]. In addition, in the present case, given a family of pair-potentials $\left(V_{a b}\right)_{a, b \in \mathcal{A}}$ of the previous type and a bounded open set $U$, it becomes possible to compute the local potential energy of a discrete measure $\nu=\left(\nu_{a}\right)_{a \in \mathcal{A}}$ as follows

$$
E_{U, r, R}(\nu)=\sum_{a, b \in \mathcal{A}} \sum_{r<|x-y|<R ; x \in U} \nu_{a}\{x\} \nu_{b}\{y\} V_{a b}(|x-y|), \quad E_{U}(\nu)=\sup _{r>0, R>0} E_{U, r}(\nu) .
$$

It can be shown that $E_{U, r, R}$ is a weak*-continuous function of $\nu$ as long as $r>0$ and $R<\infty$. Consequently $E_{U}$ is lower semicontinuous (in particular configurations with more than one atom at the same point inside $U$ have infinite local energy). The previous energy can be decomposed into the energy internal to $U$, corresponding to the pairs $x, y$ in $U$ and the interaction between $U$ and its complement $U^{c}=\mathbb{R}^{d} \backslash U$. Hence

$$
E_{U}(\nu)=E_{U}^{i n t}(\nu)+E_{U ; U^{c}}(\nu) .
$$

A real or complex valued function over $\mathrm{QD}_{\mathcal{A}}\left(\mathbb{R}^{d}\right)$ will be called cylindrical if it is continuous and if there is a bounded open set $U$ such that $F$ depends only upon the restriction of $\nu$ to $U$. Then $U$ will be set to support $F$.

Definition 1. A configuration of atoms, namely a point in $\mathrm{QD}$, is Delone if there are $0<r<$ $R<\infty$ such that in any ball of radius $r$ there is at most one atom, while in any ball of radius $R$ there is at least one atom. The family of Delone sets corresponding to the parameters $r, R$ is denoted by $\operatorname{Del}_{r, R}\left(\mathbb{R}^{d}\right)$.

Delone Set Hypothesis: The only physically relevant configuration are Delone sets.

This hypothesis is justified by two observations. First two atoms are unlikely to get too close to each other, due to the strong repulsion at short distance. Second, in a condensed phase where the atomic density is high, the attracting part of the potential makes unlikely the occurrence of some large region void of atoms. That such an hypothesis is reasonable will be discussed in the forthcoming sections. The following result is fundamental

Proposition 1 (see [2]). The space $\operatorname{Del}_{r, R}\left(\mathbb{R}^{d}\right)$ is a weak $k^{*}$-compact and translation invariant subspace of $\mathrm{QD}_{\mathcal{A}}\left(\mathbb{R}^{d}\right)$. Any translation acts as an homeomorphism. In addition, given any Delone measure $\nu$ and any open ball $B$ with diameter larger than $2 R$, the number of atoms in $B$ is bounded by

$$
\left(\frac{\operatorname{diam}(B)}{2 R}-1\right)^{d} \leq \nu(B) \leq\left(1+\frac{\operatorname{diam}(B)}{2 r}\right)^{d}
$$

Definition 2. Given $\nu \in \mathrm{QD}_{\mathcal{A}}\left(\mathbb{R}^{d}\right)$ its Hull is the weak ${ }^{*}$-closure of its orbit under the translation group. In particular, if $\nu$ is $(r, R)$-Delone $\operatorname{Hull}(\nu) \subset \operatorname{Del}_{r, R}\left(\mathbb{R}^{d}\right)$ is compact and the translation group acts by homeomorphism.

2.3. Thermal Equilibrium. Using a pair potential of the previous type, it becomes possible to describe the thermal equilibrium in a rigorous way using a local Gibbs distribution. Let $F$ be a cylindrical function supported by $U$. Then the thermal average of $F$ in $U$ is usually defined by $[27,28]$ 


$$
\mathbb{E}_{U}(F)(\nu)=\frac{1}{Z_{U}(\beta, \mu ; \nu)} \sum_{N_{a}=0}^{\infty} \prod_{a \in \mathcal{A}} \frac{e^{\beta \mu_{a} N_{a}}}{N_{a} !} \int_{x_{k_{a}} \in U} \prod_{a \in \mathcal{A}} \prod_{k_{a}=1}^{N_{a}} d x_{k_{a}} e^{-\beta\left(E_{U}^{i n t}(x)+E_{U ; U^{c}}(x, \nu)\right)} F(x)
$$

where $\beta=1 / k_{B} T$ denotes the inverse temperature measure in units of energy, $\mu=\left(\mu_{a}\right)_{a \in \mathcal{A}}$ denotes the family of chemical potentials associated with each atomic species, $x=\left(\left(x_{k_{a}}\right)_{k_{a}=0}^{N_{a}}\right)_{a \in \mathcal{A}}$ denotes the (random) positions of the atoms of each species inside $U, N_{a}$ denotes the (random) number of atoms of species $a$ inside $U$, while $\nu \in \mathrm{QD}_{\mathcal{A}}$ fixes the position of the atoms outside of $U$. As it is usual, the chemical potential is fixed by the atomic densities of each atomic species at a given temperature. Hence $\mathbb{E}_{U}$ defines a probability measure on $\mathrm{QD}_{\mathcal{A}}\left(\mathbb{R}^{d}\right)$ called (local or $U$-) Gibbs state. It is worth noticing that the kinetic contribution to the Gibbs state factorizes out and can be ignored here. It is also important to realize that since the configurations having two atoms or more on top of each other inside $U$ have an infinite energy, they have zero probability w.r.t. the local Gibbs state.

In principle, the infinite volume limit is rigorously controlled through the so-called DobrushinLanford-Ruelle equations (DLR) (see [26, 30, 27] for the DLR equations and [31, 27, 28] for the formalism with classical particle systems). Namely a Gibbs state is a probability measure $\mathbb{P}$ over $\mathrm{QD}_{\mathcal{A}}\left(\mathbb{R}^{d}\right)$ admitting $E_{U}$ as conditional probability when conditioned by the configurations outside $U$. In particular such a probability is locally absolutely continuous. It is known that, under certain conditions on the pair-potentials, the set $\mathfrak{G}$ of Gibbs states is a Choquet simplex, namely it is compact and convex. In particular $\mathfrak{G}$ admits extremal points and any Gibbs state is a unique convex combination of extremal points. The same property occurs for the subset $\mathfrak{G}_{t}$ of translation invariant Gibbs states. As it turns out, the extremal states in $\mathfrak{G}_{t}$ have two important properties:

(i) they are ergodic under the translation group

(ii) they correspond to the pure homogeneous phases

2.4. The Ergodicity Paradox. The property of extreme translation invariant Gibbs states of being ergodic under the translation group has several important consequences summarized as follows

Proposition 2 (see [2]). Let $\mathbb{P}$ be a translation invariant ergodic measure on $\mathrm{QD}_{\mathcal{A}}\left(\mathbb{R}^{d}\right)$. Then (i) there is a weakly closed subset $\Omega \subset \mathrm{QD}_{\mathcal{A}}\left(\mathbb{R}^{d}\right)$ such that for $\mathbb{P}$-almost every $\nu$ the Hull of $\nu$ coincides with $\Omega$;

(ii) If $\mathbb{P}$ is supported by the space of Delone measures, then there are $0<r \leq R<\infty$ such that $\mathbb{P}\left\{\operatorname{Del}_{r, R}\left(\mathbb{R}^{d}\right)\right\}=1$ and $\mathbb{P}\left\{\operatorname{Del}_{r^{\prime}, R^{\prime}}\left(\mathbb{R}^{d}\right)\right\}=0$ if $r^{\prime} \leq r, R^{\prime} \geq R$ and $\left(r^{\prime}, R^{\prime}\right) \neq(r, R)$.

The main problem with this approach comes from the following remark: (i) the set $\operatorname{Del}\left(\mathbb{R}^{d}\right)$ of all Delone sets is translation invariant, (ii) it is a Borel subset of $\mathrm{QD}_{\mathcal{A}}\left(\mathbb{R}^{d}\right)$ as a countable union of compact sets. Hence the ergodicity of $\mathbb{P}$ implies that the set of Delone sets has either probability zero or probability one. However it is quite standard to prove that at positive temperature, the set of configurations having either an arbitrary big hole somewhere or an arbitrary number of particles in a small ball somewhere else, has positive density. This can be done by evaluating the probability of a big hole in a finite volume. This density is very small, but it is not zero. Hence at positive temperature $\mathbb{P}\left(\operatorname{Del}\left(\mathbb{R}^{d}\right)\right)=0$ whenever $\mathbb{P}$ is a translation invariant pure phase. Consequently the Delone set Hypothesis is mathematically incorrect.

There are two ways to reconcile Ergodicity with the Delone Hypothesis. The first one is to remark that in the limit of zero temperature only the minimal energy configuration (groundstates) 
matter. If a big hole occurs, it is energetically more favorable to fill it. Similarly an accumulation of particles in a small ball is energetically unfavorable. This is expressed by the following result $[24]$

Theorem 1 (see [24]). Let the two-body potentials $V_{a b}$ be hard-core, namely there is $r>0$ such that $V_{a b}(x)=+\infty$ if $|x| \leq r$. Then, whenever the chemical potentials belong to a range allowed by the densities of each species, there is $R>r$ such that each ground-state configuration belongs to $\operatorname{Del}_{r, R}\left(\mathbb{R}^{d}\right)$.

In the previous statement, the hard-core hypothesis is artificial because it puts by hand a property that should be spontaneously satisfied by the system. So the following problem is expected to have a positive answer

Problem 1. Prove or disprove that there is a physically relevant class of rotation invariant pair potentials such that, for a high enough density of atoms, given the concentration of each atomic species, any zero temperature limit point of translation invariant Gibbs states gives probability one to $\operatorname{Del}\left(\mathbb{R}^{d}\right)$.

The second way to reconcile Ergodicity with the Delone Hypothesis is to appeal to the persistence theory [29]. Namely rare events have a very short lifetime. This is why large vacancies are not observed in liquids and solids [25]. Similarly concentration of many atoms in a small ball is essentially impossible to observe. If, however, such extreme configurations were easy to observe, then it could be possible, for instance, to force the fusion of nuclei, namely to allow for cold fusion experiments. Hence there is a problem with the Thermal Equilibrium approach: no time scale is present. Introducing the time evolution in the problem is not easy, but there should be a way to do so in order to reconcile the observations with the mathematical approach.

Problem 2. Is it possible to develop a mathematically rigorous time dependent approach in order to reconcile the Delone Hypothesis with the translation Ergodicity of Gibbs states ? More generally, is it possible to formulate and to develop a finite time approximation of an ergodic dynamical system permitting to neglect rare event with too short lifetime?

\section{The Anankeon Theory}

This Section provides few arguments, found by physicists, leading to the concept of anankeon.

3.1. Glasses, Frustration, Local Stress. It is a known fact of practice that most materials, in particular metals, have a tendency to crystallize. Hence producing glasses with metals has been a real challenge (see [37] for a review). The earliest technique was melt quenching, namely an ultrafast cooling with cooling rate of $10^{5}-10^{6} \mathrm{~K} / \mathrm{s}$. This technique limits sample to thin ribbons. Other techniques were developed later. Eventually a compound with low cooling rate was produced by Inoue et. al. (see [12]). Later the class of vitalloy was produced by Johnson et. al. (see [13]) and is nowadays the most studied class of bulk metallic glasses. Vitalloy can be casted in large samples of several centimeters. It was quickly realized that these new materials had exceptional mechanical properties, boosting the subject in the front stage of material science. Thanks to the theoretical modeling of spin-glass the concept of frustration emerged as a fundamental aspect of glassy states [35]. It was slowly understood that such a frustration occurs in metallic glasses whenever several atomic species with different atomic radii where mixed together in such proportions as to force atoms of different size to be close to each other. In this way the number of small clusters that can be formed in the melt is getting larger $[6,18]$ and crystallization becomes unlikely. 
Another ingredient that should be included, advocated for a long time by Egami em et al., is the local stress exercised by neighboring atoms on a single one. For indeed, as can be shown by a Thomas-Fermi approximation [32,34], atoms are not exactly hard ball. They can be squeezed a little, however big is their resistance to pressure. This leads to associating with each atom a stress tensor $\sigma_{i}$, by adding the contributions of forces acted upon by the neighboring atoms. An important observation, made through numerical simulations using molecular dynamics, is the following:

Egami-Srolovitz Principle: In the liquid phase, the atomic stress tensors behave like independent identically distributed random Gaussian variables [33]

More precisely, a stress tensor $\sigma$ is a $3 \times 3$ real symmetric matrix. It is usually decomposed into its trace part $p=(1 / 3) \operatorname{Tr}(\sigma)$, called the pressure, and its traceless part $\widehat{\tau}=\sigma-p$ called the deviatoric stress. Then the parameter $\tau$, defined by $\tau^{2}=(3 / 2) \operatorname{Tr}\left(\widehat{\sigma}^{2}\right)$, is called the von Mises stress. The atomic stress distribution is given by [33] the 6-dimensional integral

$$
\operatorname{Prob}\left\{\sigma_{i} \in A\right\}=\frac{1}{Z} \int_{A} e^{-\beta\left(p^{2} / 2 B+\tau^{2} / 2 G\right)} d \sigma .
$$

where $A \subset M_{3}^{s}(\mathbb{R})$ is any Borel set, $d \sigma$ denotes the usual Haar measure on $M_{3}^{s}(\mathbb{R})$ and $Z$ is the normalization constant. The physical parameters $B, G$ are called the bulk modulus and the shear modulus respectively. This expression must, however, be renormalized at lower temperature, when approaching the glass transition. This renormalization, proposed by Eshelby [8], is the analog for continuum mechanics to the Clausius-Mossotti formula for dielectric. Namely the long distance propagation of stress, due to the elasticity equation, creates a local effective stress field on the local atom which can be summarized as a modification of the values of $B, G$. This numerical study suggests that, in the liquid phase, the stress degrees of freedom are dominant and behave like a free gas at equilibrium, in complete analogy with the Einstein theory of phonons in crystals. In particular, the heat capacity becomes elementary to compute and behaves like a constant at high temperature: this is the origin of the Law of Dulong-Petit, confirmed by experiments [4] (see Fig. 1 and eq. (1) in Section 1.3). The main question is to give this finding an explanation from first principle.

3.2. Anankeon. The intuitive reason why the local stress is actually random in the liquid phase can be seen by imagining what the life of the local atom should be. The atom can never find a position that minimizes all pair potential energy and this is the result of the local frustration. Even in the ideal situation, for which the local cluster is made of identical atoms localized on the center and the vertices of a perfect icosahedron, the distance between the central atom to the vertex-ones is $5 \%$ less than between two vertex-atoms, meaning that it is not possible to minimize all pair potentials simultaneously. The random thermal motion then intervenes to move atoms around in search for a better position. Hence the corresponding stress tensor felt by each atom is constantly varying under the stress of circumstances, a concept that can be translated by the word $\alpha \nu \alpha \gamma \kappa \epsilon \iota \alpha$ (anagkeia) in Greek.

There is a a character of the Greek mythology that could fit with this concept, the goddess Ananke, whose name comes from the greek word anagkeia. Ananke was representing a power above all including the Gods of the Greek Mythology. It expresses the concepts of "force, constraint, necessity" and from there it also means "fate, destiny" to lead to the concepts of compulsion, torture (see the entry Ananke in Wikipedia). From this, the word anankeon can be coined to describe the dominant degrees of freedom taking place in the liquid phase [1]. What are these anankeons? It is the purpose of what follows to make this concept more precise. In the 
physics literature focussing on this problem, these degrees of freedom are also called topological, meaning that the local atomic arrangement, also called local clusters, or local patches in tiling theory, is modified. Another way to put it is to describe the association of atoms in terms of bonds in a way similar to chemical bonds. The movement of atoms corresponds to a random change in the bonding between atoms.

\section{Topology and Combinatoric of the Space of Delone Sets}

It will be argued that a Voronoi construction [44] (also called Dirichlet tesselation) on the set of configurations is the right mathematical tool liable to describe anankeons. In what follows, the Delone set Hypothesis is assumed to hold and all atomic configurations will be chosen in

$\operatorname{Del}_{r, R}\left(\mathbb{R}^{d}\right)$ which will be denote by Del. For simplicity the specification of various atomic species and of their relative concentration will be omitted here. Then $\mathrm{Del}^{0}$ will denote the set of such configurations having one atom at the origin. This Section is a summary of a work in preparation [38]. In particular all Theorems and other claims will be proved there. The author suspects that several of these results are already known but did not find yet the corresponding references.

4.1. Delaunay Triangulation. The Voronoi tiling point of view, was already used by Bernal [3]. Given a discrete Delone set $\mathcal{L} \subset \mathbb{R}^{d}$, define the Voronoi cell $V_{x}$ of a point $x \in \mathcal{L}$ (called atoms here) as the set of points in the space closer to $x$ than to any other atom in $\mathcal{L}$. Since $\mathcal{L}$ is Delone, say with parameters $(r, R)$, it follows that $V_{x}$ is an open convex polytope containing the open ball $B(x ; r)$ and contained in the ball $B(x ; R)$. The Voronoi tile at $x$ is just the closure $T_{x}=\overline{V_{x}}$. Two distinct Voronoi cells have no point in common, while two distinct Voronoi tiles may intersect only on a common face. Two distinct atoms $x, y \in \mathcal{L}$ will be called nearest neighbors whenever their Voronoi tiles are touching along a common face of codimension one (namely of dimension $d-1)$. The set $\mathcal{E}$ of pairs $e=(x, y)$ of nearest neighboring atoms, called edge or bonds, defines with $\mathcal{L}$ a graph $\mathcal{G}=(\mathcal{L}, \mathcal{E})$ which will be called the Delone graph. It is known in the literature as the Delaunay Triangulation [40]. It plays the role of a dual lattice. This graph is simple (between any two vertices there is at most one edge), connected (any two vertices can be linked by a finite path, namely a sequence of edges touching by a common vertex). Moreover the length of any edge lies between $2 r$ and $2 R$. Hence the graph distance $d_{\mathcal{G}}(x, y)$ between two atoms, defined as the length of the shortest path linking $x$ to $y$, is equivalent to the Euclidean distance.

4.2. Voronoi Points, Delaunay Triangulation and the Empty Sphere Property. In this work the name Voronoi point will be given to a vertex of one of the Voronoi tiles, namely a face of dimension zero (see Fig. 3). An atomic neighbor (or a-neighbor) of a Voronoi point is an atom with Voronoi tile containing this point. For example, in Fig. 3, the Voronoi point indicated on the left has 4-a-neighbours, while a small deformation of the atomic positions, as seen on the right shows that this Voronoi point has split and each of the new Voronoi points has exactly 3-a-neighbors (generic situation). It is important to remark that atomic sites have a material existence in a solid, while Voronoi points are just a convenient mathematical concept that does not correspond to any material point in the solid.

The following result is classic and goes back to [40]

Theorem 2. Let $\mathcal{L} \in \operatorname{Del}^{0}$.

(i) Any Voronoi points of $\mathcal{L}$ has at least $d+1$ a-neighbors. It belongs to the interior of the convex hull of its a-neighbors. Generically, in $\mathrm{Del}^{0}$, all Voronoi points have exactly $d+1$ neighbors.

(ii) Empty sphere property: The set of a-neighbors of a Voronoi point $y$ of $\mathcal{L}$ is contained in a sphere centered at $y$ the interior of which contains no other atoms. Conversely, any sphere 
in $\mathbb{R}^{d}$ defined by at least $d+1$ atoms of $\mathcal{L}$ which does not contained atoms in its interior admits a Voronoi point at its center.

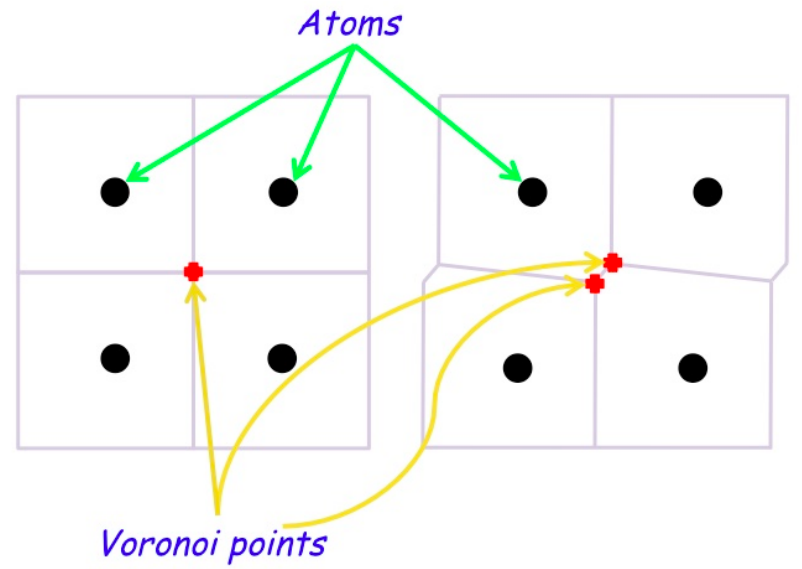

Figure 3

A Voronoi point will be called simple whenever it has exactly $d+1$ a-neighbors. Generically, all Voronoi points of $\mathcal{L}$ are simple. Hence they are at the center of an empty sphere and the corresponding atoms give elementary $d$-simplex (triangles in $2 D$ and tetrahedra in $3 D$ ) making up a triangulation of the space, called the Delaunay triangulation.

4.3. Graph Balls and Local Topology. A graph ball is a subgraph $\mathcal{G}(x, n)=(\mathcal{V}(x, n), \mathcal{E}(x, n))$, where $\mathcal{V}(x, n)$ is the set of all atoms at a graph distance less than or equal to $n \in \mathbb{N}$ from $x$, and $\mathcal{E}(x, n)$ is the set of all edges $(y, z) \in \mathcal{E}$ such that $y, z \in \mathcal{V}(x, n)$. Then $x$ is the center and $n$ the radius of this graph ball. The support of a graph ball is the union of Voronoi tiles centered at its vertices.

Two graphs $\mathcal{G}, \mathcal{G}^{\prime}$ are called isomorphic if there are bijective maps $\phi^{v}: \mathcal{V} \rightarrow \mathcal{V}^{\prime}$ and $\phi^{e}: \mathcal{E} \rightarrow \mathcal{E}^{\prime}$ compatible with the edge-boundary, namely the vertices linked by the image $\phi^{e}(e)$ (the boundary) of an edge $e$ is the $\phi^{v}$-image of the vertices liked by $e$. Equivalently a graph isomorphism is an isometry of its vertex set endowed with the graph distance.

A local patch is an isomorphism class of graph balls. In particular all such balls have the same radius. In this sense a local patch is topological, because it is likely to be insensitive to small move of the corresponding atoms. It is essential to remark that

Proposition 3. The number of local patches of given radius is finite namely the Delone Graphs have Finite Local Complexity.

In other words, when the atoms are moving a bit, the corresponding Delone graph is deformed, but its isomorphism class does not change.

4.4. Alloys. If $\mathcal{L}$ represent the position of the atoms in an alloy with more than one species, the Voronoi construction can be done provided the Euclidean distance is replaced by a local metric taking care of the different atomic radii. Namely $V_{x}$ is defined as the set of points $z \in \mathbb{R}^{d}$ such that $\|z-x\| / a_{x}<\|z-y\| / a_{y}$ for all $y \in \mathcal{L}$ distinct from $x$, where $a_{x}$ denotes the atomic radius of the atom $x$. Then the Voronoi cells are curved polytopes, namely their faces are made of pieces of spheres. But all the combinatorial aspect described here will persist. 
4.5. Acceptance Domains. Given a local patch $\mathcal{P}$ of radius $n$, its acceptance domain $D(\mathcal{P})$ is the set of all Delone sets in $\mathrm{Del}^{0}$ with graph ball of radius $n$, centered at the origin, given by $\mathcal{P}$. Given $n \in \mathbb{N}$, the family of all acceptance domains of local patches of radius $n$ is a partition of of $\mathrm{Del}^{0}$. A local patch will be called generic whenever its acceptance domain is open.

Theorem 3 ([38]). A local patch $\mathcal{P}$ of radius $n$ is generic if and only if the support of the $n$-graph ball centered at the origin of any atomic configuration in its acceptance domain contains only simple Voronoi points.

The closure of an open acceptance domain will be called a domain of contiguity

Theorem 4 ([38]). The set of domains of contiguity of patches of radius $n$ is a finite cover of $\mathrm{Del}^{0}$.

In a sense the family of generic patches of given radius generates a finite tiling of $\mathrm{Del}^{0}$, if the tiles are the domain of contiguity while their interiors are the open acceptance domains. Moreover passing from $n$ to $n+1$ gives a refinement of this tiling, namely any contiguity domain of radius $n$ is tiled by the contiguity domains of radius $n+1$ it contains. The following shows that genericity coincides with full probability with respect to any Gibbs state,

Theorem 5 ([38]). Given $\mathbb{P}$ a locally absolutely continuous probability measure on $\mathrm{Del}^{0}$ the boundary of a contiguity domain has zero probability.

4.6. Graph of Contiguity. Given $n \in \mathbb{N}$, two contiguity domains of radius $n$ are contiguous if their intersection contains a subset of co-dimension one. Then the graph of contiguity $\mathfrak{G}_{n}=$ $\left(\mathfrak{V}_{n}, \mathfrak{E}_{n}\right)$ is defined as the graph with set of vertices $\mathfrak{V}_{n}$ given by all domain of contiguity of radius $n$ (or equivalently by the set of generic patches) and edge set $\mathfrak{E}_{n}$ given by contiguous pair of domains of contiguity in $\mathfrak{V}_{n}$. As it turns out

Theorem 6 ([38]). $\mathfrak{G}_{n}$ is a finite, simple and connected graph. The number of its vertices increases exponential fast with $n$.

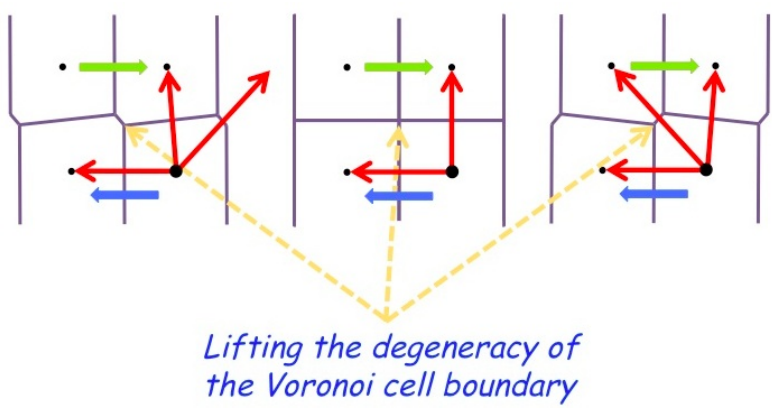

FiguRE 4. Pachner move in dimension 2: a $2 \leftrightarrow 2$ Voronoi point collision

4.7. Contiguity and Pachner Moves. The previous description makes sense from a mathematical standpoint. But it looks extremely complicate at first sight, since the space $\mathrm{Del}^{0}$ is infinite dimensional: indeed, the restriction of $\mathrm{Del}^{0}$ to any bounded domain $U$ can be seen as the disjoint union of a family of open sets in $\mathbb{R}^{n d}$, where $n$ denotes the number of atoms inside $U$. It is actually simple to describe a transition from a domain of contiguity to a contiguous one. For indeed, such a move requires only one local change in the Delone set. To express this fact 
more precisely let $n \in \mathbb{N}$ be chosen as the radius of patches. Then a generic atomic configuration belonging to the intersection $\mathcal{T}_{0} \cap \mathcal{T}_{1}$ of two contiguous domains of contiguity is one with only one non simple Voronoi point within the ball of radius $n$ centered at the origin. In addition, this Voronoi points admits exactly $d+2$ a-neighbors. In particular:

(i) in dimension 2, this Voronoi point admits 4 atomic neighbors (see Fig. 4),

(ii) in dimension 3 it has 5 atomic neighbors (see Fig. 5).
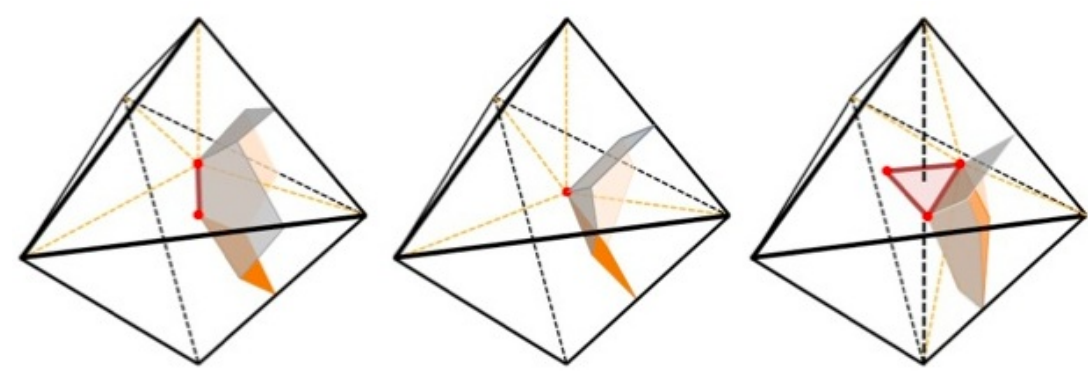

FiguRE 5. Pachner move in dimension 3: a $2 \leftrightarrow 3$ Voronoi point collision

As soon as the configuration moves away from the $\mathcal{T}_{0} \cap \mathcal{T}_{1}$, this nongeneric Voronoi point splits into a family of generic ones and this splitting is known in triangulation theory used in Geometry as a Pachner move [43] or bistellar flips. More precisely (see Fig. 4 and Fig. 5),

(a) in dimension 2, two generic Voronoi points collide at the transition to give two other ones;

(b) in dimension 3, two generic Voronoi points collide to give three other ones or vice-versa.

(c) in higher dimension $l$ Voronoi points collide at the transition to give $m=d+2-l$ other ones for $2 \leq l \leq d$.

\section{Applications to Material Science}

The previous mathematical program can be applied in practical circumstances. Pachner moves, seen as collisions of Voronoi points leads to several practical consequences. In a sense the anankeon can be seen as the degree of freedom associated with the motion of Delaunay simplex, (triangle in dimension 2, tetrahedron in dimension 3) formed by the $d+1$ atoms around the Voronoi point. Hence an anankeon can be labeled by this Voronoi point.

5.1. Pachner Moves in Materials. The previous results should apply in real material, apart from taking energy considerations into account. A Pachner move corresponds to a mechanical motion of the atomic configuration passing through a saddle point of the potential energy. In particular if the atomic configuration corresponds to a point of the energy surface close enough to the saddle point, it looks almost like the perfect nongeneric Voronoi point. In practice it means that when several Voronoi points are very close to each other, then the configuration is physically equivalent to a nongeneric unique Voronoi point. In the scheme described in Section 4.5, it means that the boundary of a domain of continuity is actually thicken by the energetic considerations. For this reason the number of atoms involved in a Pachner move is likely to be a random variable. This is what has been observed recently in the Egami group [49] (see Figure 6).

It is remarkable that this distribution is Poissonian with average 5 as predicted by the Pachner theory in dimension 3. 


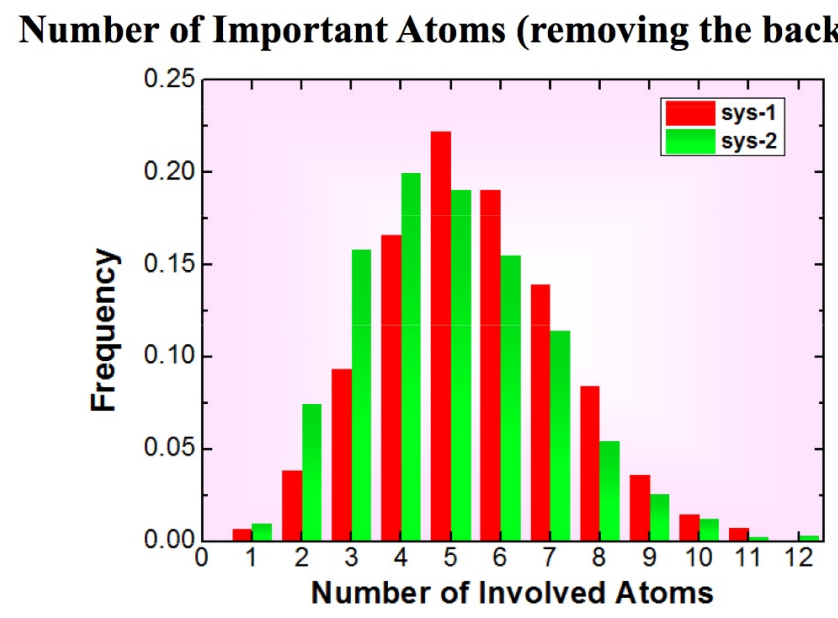

Figure 6. Distribution of the number of atoms involved in a Pachner move [49]

5.2. Pachner Moves and STZ. The shear transformation zone theory (STZ) proposed by Langer et al. $[9,10,15,16,17]$, consists simply in remarking that indeed such Pachner moves occur in glasses when submitted to a strong shear. In the glass phase, however, the atoms are blocked in their position, so that the dominant degree of freedom is provided by the phonons only. However, if a shear is applied, first the solid reacts elastically. But beyond some critical value of the shear, the deformation becomes irreversible. At the atomic scale, this can be interpreted as one Pachner move somewhere and the numerical simulations show that such a move does not come alone, it is followed by a cascade of moves in the vicinity. In other words a small region about the germ behaves like a liquid, and this is exactly what an STZ is. The spatial distribution of such STZ is random, presumably Poissonian with a density depending upon the applied shear. This is exactly the hypothesis made in the STZ theory. Each Pachner moved is polarized, as can be figured out from Figures 4 and 5. In $2 D$ a Pachner moves corresponds the a switch of a diagonal bond from left to right or vice-versa. In $3 D$ it corresponds to a collision of 2 Voronoi points giving 3 or vice-versa. In both cases, there is a change between two types of configurations, each of which being called a polarization. Then the local parameter is provided by the numbers $N_{ \pm}$of each of these varieties of groups Voronoi points in the STZ. This number is a random variable the time evolution of which is assumed to be Markovian, namely it follows a Master Equation. As it turns out, this effect can be added to the equation of elasticity, including the temperature effects. Eliminating the parameters $N_{ \pm}$from them leads to a non linear partial differential equation which describe accurately the plasticity region in the strain-stress relation for a metallic glass [10]. Using this equation it becomes possible to simulate numerically in a realistic way a fracture of the material [20].

5.3. Atomic Scale Markov Process. In order to describe the anankeon dynamics, the previous considerations leads to represent it by a Markov process on the graph of contiguity $\mathfrak{G}_{n}$. The radius $n$ is simply a measure of the volume of the region under consideration. A Markov process represents the dynamics of a random walker moving on the set of vertices of the graph through the edges (standard references are [47, 48] for discrete time and [45, 46] for continuous time). Since $\mathfrak{G}_{n}$ is a finite graph, a Markov process can be equivalently described by its generator $\mathfrak{L}_{n}$, 
namely a linear operator acting of the linear space $\mathcal{H}_{n}$ of complex valued functions defined on $\mathfrak{V}_{n}$, as follows

$$
\mathfrak{L}_{n}(f)(\mathcal{P})=\frac{1}{T_{\mathcal{P}}} \sum_{\mathcal{Q} \sim \mathcal{P}} \mathbb{P}_{\mathcal{L} \leftarrow \mathcal{P}}(f(\mathcal{P})-f(\mathcal{Q})) .
$$

in such an expression, $\mathcal{P}, \mathcal{Q}$ label patches of radius $n$ which are contiguous, and $\mathbb{P}_{\mathcal{Q} \leftarrow \mathcal{P}}$ represent the probability to jump from $\mathcal{P}$ to $\mathcal{Q}$. Moreover $T_{\mathcal{P}}$ represents the waiting time of the random walker at the patch $\mathcal{P}$. Since this Markov process is supposed to define the thermal equilibrium motion, the jump probability is given by a Gibbs factor of the from

$$
\mathbb{P}_{Q \leftarrow \mathcal{P}}=\frac{1}{z} e^{-\beta(F(\mathcal{Q})-F(\mathcal{P}))}
$$

where $F(\mathcal{P})$ represents the free energy of the configurations represented by $\mathcal{P}$, while $\mathcal{Z}$ is a normalization constant ensuring that the sum over $\mathcal{Q}$ of the r.h.s. equals 1 . On the other hand, the waiting time is provided by an Arrhenius law and depends only of the potential barrier $W(\mathcal{P} \rightarrow \mathcal{Q})$ between the initial configuration and the saddle points during Pachner moves

$$
T(\mathcal{P})=e^{\beta W(\mathcal{P} \rightarrow Q)} .
$$

Numerical simulations based on molecular dynamics permit to estimate precisely the previous parameters $[41,42]$ and, in principle, to test the validity of such an approach. However, for the previous description to be realistic, the atomic stress degrees of freedom must be introduced in the Markov modeling.

5.4. Viscosity and Glass Transition. Once the Markov dynamics is defined and tested, it becomes possible to compute the viscosity. It is defined as the linear response to an applied shear in terms of the time derivative of the strain. Thanks to the Green-Kubo formula, it is also equal to the stress-stress correlation function. As the temperature decreases, the thermal activation moving the atoms around becomes less effective. In particular given some shear, it takes more Pachner moves to achieve the same amount of strain variation. Hence the typical time scale involved in the viscosity (called the Maxwell time) becomes much larger than the time scale involved in the definition of the Markov process. The glass transition is achieved when the the Maxwell time becomes so large as to make it impossible to observe. Using this principle it becomes possible to give a formula for the temperature of the glass transition [39] fitting with experimental datas.

\section{Geometry on the Space of Delone Configurations}

This Section is conjectural and proposes a program liable to define on the compact space $\operatorname{Del}^{0}$ a geometry, namely a metric, liable to describe, in analogy with General Relativity, the dynamics of a condensed material in terms of a geodesic flow with respect to this metric structure.

6.1. Infinite Volume Limit. In Section 5.3 a Markov process is proposed as a modeling of the time evolution of a liquid or glassy material. This Markov process, though, is defined on graph balls of a fixed finite radius $n \in \mathbb{N}$. The question remains whether the sequence of such process converges to some limit in the limit where the radius $n$ tends to infinity. This is an entirely open problem so far. However, there are indications that it could be possible to prove the convergence. One strategy could be to use the martingale convergence Theorem $[57,58]$. It is a technique used elsewhere to prove the existence of a diffusion process on fractals like the Sierpinski carpet [50]. If so, then the generator $\mathfrak{L}$ of this limiting Markov process will acts on the 
entire $\mathrm{Del}^{0}$ space as a Dirichlet form $[51,52,59]$. Another strategy could be to use convergence Theorem for Markov semigroups on $C^{*}$-algebras [53].

6.2. Dirichlet Forms and Gradient Operators. It is important though that the Markov process have the so-called Feller property, namely that the image of a continuous function on $\mathrm{Del}^{0}$ by the process stays continuous. If so, this Markov process should be represented as a Markov semigroup on the $C^{*}$-algebra $\mathcal{A}=\mathcal{C}\left(\mathrm{Del}^{0}\right)$, namely the space of continuous functions on $\mathrm{Del}^{0}$. More is needed though: namely the domain of $\mathfrak{L}$ should contain a dense $*$-subalgebra of $\mathcal{A}$. In a such a case, an extension of the Lindblad Theorem leads to the following: there are

(i) a $C^{*}$-right $\mathcal{A}$-module $\mathcal{E}$

(ii) a $*$-representation $\pi: \mathcal{A} \rightarrow \operatorname{End}(\mathcal{E})$

(iii) a linear map $\partial: \mathcal{C}\left(\mathrm{Del}^{0}\right) \rightarrow \mathcal{E}$

such that, for every $f, g \in \mathcal{A}$

$$
\mathfrak{L}\left(f^{*} g\right)-f^{*} \mathfrak{L}(g)-\mathfrak{L}\left(f^{*}\right) g=\langle\partial f \mid \partial g\rangle_{\mathcal{E}}
$$

and that $\partial$ becomes a derivation, namely

$$
\partial(f g)=\partial(f) g+\pi(f) \partial(g) .
$$

In other words, $\partial$ is a candidate for a generalized version of the gradient operator. Since the inner product $\langle\cdot \mid \cdot\rangle_{\mathcal{E}}$ takes on values in $\mathcal{A}=\mathcal{C}\left(\mathrm{Del}^{0}\right)$, it follows that

$$
\|f\|_{\text {Lip }} \stackrel{\text { def }}{=} \sup _{\nu \in \operatorname{Del}^{0}}\left|\langle\partial f \mid \partial f\rangle_{\mathcal{E}}(\nu)\right|^{1 / 2}
$$

plays the role of a a Lipshitz norm. Hence, by the Kantorovich duality [61, 62] theory, it is likely to define a metric on $\mathrm{Del}^{0}$ through the formula

$$
d_{\mathfrak{L}}\left(\nu, \nu^{\prime}\right)=\sup \left\{\mid f(\nu)-f\left(\nu^{\prime} \mid ;\|f\|_{\text {Lip }} \leq 1\right\} .\right.
$$

Problem 3. Prove that all conditions are fulfilled to make sure that the metric $d_{\mathfrak{L}}$ is well defined and generates the weak ${ }^{*}$-topology on $\mathrm{Del}^{0}$.

Such a problem has already been investigated in full generality by Rieffel in the context of Quantum Metric Spaces [65, 66, 67, 68, 63], providing a criterion to answer positively to this question.

6.3. The Curvature Problem. The previous construction, if it works, leads to a structure of compact metric space on $\mathrm{Del}^{0}$ generated by the dynamics describing the time evolution for glasses and liquids. One important question is to check whether such a structure share with Riemannian manifolds a notion of curvature. In Noncommutative Geometry, thanks to the work of I. Palmer [64], a Riemannian structure can be defined on any compact metric space using the concept of spectral triple [55]. However since the Hausdorff dimension of $\mathrm{Del}^{0}$ is infinite, the construction cannot be used very far. In particular it is likely that a Connes state, namely a Dixmier trace, does not exist. Hence one can only expect a KMS state with respect to the Dirac operator associated with the spectral triple.

Problem 4. Elucidate the meaning of the KMS condition produced in this way. Can one see the Connes state as the equilibrium invariant state associated with the Markov dynamics generated by $\mathfrak{L}$ ? 
In addition, the definition of the curvature has not yet reached a consensus in Noncommutative Geometry [56]. So, it might be necessary to come back to the usual approach in Metric Geometry $[60,54]$. The celebrated Gromov Theorem asserts that the set of Riemannian manifolds with uniformly bounded volume, curvature and injectivity radius, is precompact in the HausdorffGromov metric topology. It raises the question of what is a limit point in this topology. Examples are given by Alexandrov spaces, namely path-metric spaces in which geodesic exist between any pair of points, the concept of angle between two geodesic starting at the same point is welldefined, and in which a concept of scalar curvature exists [54]. The curvature is obtained from comparing geodesic triangles in this space with isometric ones in model Riemannian manifolds with constant curvature [54].

Problem 5. If the construction of Section 6.2 can be made, is it possible to see $\mathrm{Del}^{0}$ as an Alexandrov space? If yes, is there an interpretation of the curvature in terms of the Continuum Mechanics of the condensed material under scrutiny?

Such a relation is exactly given by the Einstein equation in General relativity, linking the gravitational force to the Geometry of the Universe. This series of question suggests to look at whether such an interpretation is likely to hold in the description of a condensed material.

\section{REFERENCES}

\section{Introduction}

[1] The name anankeon used in these notes is the result of an exchange of emails between Takeshi Egami and the author in the Fall 2012. It has been used for the first time in a publication in [7].

[2] J. Bellissard, D. Hermmann, M. Zarrouati, "Hull of Aperiodic Solids and Gap labeling Theorems", in Directions in Mathematical Quasicrystals, CRM Monograph Series, 13, 207-259, M.B. Baake \& R.V. Moody Eds., AMS Providence, (2000).

[3] J. D. Bernal, "The Bakerian Lecture, 1962. The Structure of Liquids", Proc. R. Soc. A, 280, (1984), 299-322, and references therein.

[4] H. S. Chen, D. Turnbull, "Evidence of a Glass-Liquid Transition in a Gold, Germanium Silicon Alloy", J. Chem. Phys., 48, 2560-2571, (1968).

[5] S.-P. Chen, T. Egami, V. Vitek, Phys. Rev. B, 37, (1988), 2440-2449.

[6] T. Egami, Y. Waseda, "Atomic size effect on the formability of metallic glasses", J Non-Cryst Solids, 64, (1984), 113-134.

[7] T. Egami, "Elementary Excitation and Energy Landscape in Simple Liquids", Mod. Phys. Lett. B, 28, (2014), 1430006 1-19.

[8] J. D. Eshelby, "The Determination of the Elastic Field of an Ellipsoidal Inclusion, and Related Problems", Proc. R. Soc. London, 241, (1957), 376-396.

[9] M. L. Falk, J. S. Langer, "Dynamics of viscoplastic deformation in amorphous solids", Phys. Rev. E, 57, (1998), 7192-7205.

[10] M. L. Falk, J. S. Langer, L. Pechenik, "Thermal effects in the shear-transformation-zone theory of amorphous plasticity: Comparisons to metallic glass data", Phys. Rev. E, 70, (2004), 011507.

[11] J. P. Hansen, I. R. McDonald, Theory of Simple Liquids, Second Edition, Academic Press, London (1990).

[12] A. Inoue, "Stabilization of metallic supercooled liquid and bulk amorphous alloys", Acta Mater., 48, (2000), 279-306.

[13] W. L. Johnson, "Bulk Glass-Forming Metallic Alloys: Science and Technology", (1998 Material Research Society Medal Award Lecture, Presented at Symposium), in MRS Proceedings, Volume 554, (1998); "Bulk glass-forming metallic alloys: science and technology", Mater. Res. Soc. Bull., 24, (1999), 42-56.

[14] L. D. Landau, E, M. Lifshitz, Theory of Elasticity, Volume 7 of Course on Theoretical Physics, 3rd Edition, reviewed and enlarged by E, M. Lifshitz, A. M. Kosevitch and L. P. Pitaevskii,1986, ButterworthHeinemann Linacre House Pub., Oxford, 1999. 
[15] J. S. Langer, L. Pechenik, "Dynamics of shear-transformation zones in amorphous plasticity: Energetic constraints in a minimal theory", Phys. Rev. E, 68, (2003), 061507.

[16] J. S. Langer, "Dynamics of shear-transformation zones in amorphous plasticity: Formulation in terms of an effective disorder temperature", Phys. Rev. E, 70, (2004), 041502.

[17] J. S. Langer, "Shear-transformation-zone theory of deformation in metallic glasses", Scripta Materialia, 54, (2006), 375-379.

[18] D. B. Miracle, "A structural model for metallic glasses", Nature Mater., 3, 697-702, (2004).

[19] C. H. Rycroft, E. Bouchbinder, "Fracture Toughness of Metallic Glasses: Annealing-Induced Embrittlement", Phys. Rev. Letters, 109, (2012), 194301:1-5.

[20] C. H. Rycroft, F. Gibou, "Simulations of a stretching bar using plasticity model from the shear transformation zone theory", J. Comp. Phys., 231, (2012), 2155-2179.

[21] S. A. Silling, "Reformulation of elasticity theory for discontinuities and long-range forces", J. Mech. Phys. Solids, 48, (2000), 175-209.

[22] S. A. Silling, , M. Epton, O. Weckner, J. Xiu, E. Askari, 'Peridynamics states and constitutive modeling', J. Elasticity, 88, (2007), 151-184.

[23] S. A. Silling, R. B. Lehoucq, "Convergence of Peridynamics to Classical Elasticity Theory", J. Elasticity, 93, (2008), 13-37.

\section{Why Delone Sets?}

[24] J. Bellissard, C. Radin, S. Shlosman, "The characterization of ground states", J. Phys. A: Math. Theor., 43, (2010), 305001.

[25] C. H. Bennett, P. Chaudhari, P. Moruzzi, P. J. Steinhardt, "On the stability of vacancy and vacancy clusters in amorphous solids", Phil. Mag. A40, (1979), 485-495.

[26] R. L. Dobrushin, "Gibbsian Random Fields for Lattice Systems with Pair Interactions", Funct. Anal. Appl., 2, (1968), 291-301.

[27] O. E. Lanford III, Entropy and equilibrium states in classical Statistical Mechanics", in Statistical Mechanics and Mathematical Problems, Lecture Notes in Physics Volume 20, 1973, pp 1-113.

[28] O. E. Lanford III, " Time evolution of large classical systems", in Dynamical systems, theory and application, , Lecture Notes in Physics Volume 38, 1974, pp 1-111.

[29] S. Majumdar, "Persistence in Nonequilibrium Systems", Curr. Sci., 77, (1999), 370-375, accessible on arXiv cond-mat/9907407.

[30] D. Ruelle, David Statistical mechanics: Rigorous results, W. A. Benjamin, Inc., New York-Amsterdam (1969).

[31] D. Ruelle, "Superstable interactions in classical statistical mechanics", Comm. Math. Phys., 18, (1970), 127-159.

\section{The Anankeon Theory}

[32] R. Benguria, E. H. Lieb, "The positivity of the pressure in Thomas-Fermi theory", Comm. Math. Phys., 63, 193-218.

[33] T. Egami, D. Srolovitz, "Local structural fluctuations in amorphous and liquid metals: a simple theory of the glass transition", J. Phys. F: Met. Phys., 12, (1982), 2141-2163.

[34] E. H. Lieb, "Thomas-Fermi and related theories of atoms and molecules", Rev. Modern Phys., 53, (1981), 603-641; "Erratum", Rev. Modern Phys., 54, (1982), 311.

[35] M. Mézard, G. Parisi, M. A. Virasoro, Spin glass theory and beyond, World Scientific, Singapore, (1987).

[36] K. R. Parthasarathy, Probability measures on metric spaces, American Mathematical Soc., 1967.

[37] W. H. Wang, C. Dong, C. H. Shek, "Bulk metallic glasses", Mat. Sc. Engin. R, 44, (2004), 45-89.

\section{Topology and Combinatoric of the Space of Delone Sets}

[38] J. Bellissard, paper in preparation.

[39] T. Egami, S. J. Poon, Z. Zhang, V. Keppens, "Glass transition in metallic glasses: A microscopic model of topological fluctuations in the bonding network", Phys. Rev. B, 76, (2007), 024203, 1-6.

[40] B. Delaunay, "Sur la sphère vide. A la mémoire de Georges Voronoï", Bull Acad. Sci. URSS, 6, (1934), 793-800.

[41] T. Iwashita, T. Egami, "Atomic mechanism of flow in simple liquids under shear", Phys. Rev. Lett., 108, (2012), 196001 1-4. 
[42] T. Iwashita, D. M. Nicholson, T. Egami, "Elementary Excitations and Crossover Phenomenon in Liquids", Phys. Rev. Lett., 110, (2013), 205504, 1-5.

[43] U. Pachner, "P. L. homeomorphic manifolds are equivalent by elementary shellings", European J. Combin., 12, (1991), 129-145.

[44] G. Voronoi, "Nouvelles applications des paramètres continus à la théorie des formes quadratiques", $J$. Reine Angew. Math., 133, (1908), 97-178.

\section{Applications to Material Science}

[45] R. M. Blumenthal, R. K. Getoor, Markov processes and potential theory, Monographs in Pure and Applied Mathematics, Academic Press, New-York, (1968), reprint by Elsevier, (2011).

[46] E. Çinlar, Probability and Stochastics, see Chapt. IX, Springer, (2011).

[47] J. R. Norris, Markov Chains, Cambridge University Press, (1998).

[48] D. W. Stroock, An Introduction to Markov Processes, Springer, (2005).

[49] Y. Fan, T. Iwashita, T. Egami, , "How Thermally Induced Deformation Starts in Metallic Glass", Nature Communications, in press (2014).

\section{Geometry on the Space of Delone Configurations}

[50] M. T. Barlow, R. F. Bass, "The construction of Brownian motion on the Sierpinski carpet", Ann. I.H.P., 25, (1989), 225-257.

[51] A. Beurling, J. Deny, "Espaces de Dirichlet. I. Le cas élémentaire", (French) Acta Math., 99, (1958), 203-224.

[52] A. Beurling, J. Deny, "Dirichlet spaces", Proc. Nat. Acad. Sci. U.S.A., 45, (1959), 208-215.

[53] O. Bratteli, D. W. Robinson, Operator Algebras and Quantum Statistical Mechanics, Vol. 2, SpringerVerlag, 2nd ed. (1997).

[54] D. Burago, Y. Burago, S. Ivanov, A Course in Metric Geometry, Graduate Studies in Mathematics, 33. American Mathematical Society, Providence, RI, 2001.

[55] A. Connes, Noncommutative Geometry, Academic Press, (1995).

[56] A. Connes, H. Moscovici, "Modular Curvature for Noncommutative Two-Tori", arXiv:1110.3500, (2011).

[57] J. L. Doob, "Regularity properties of certain families of chance variables", Trans. Amer. Math. Soc., 47, (1940), 455-486.

[58] J. L. Doob, Stochastic processes, John Wiley \& Sons, Inc., New York; Chapman \& Hall, Limited, London, (1953).

[59] M. Fukushima, Dirichlet Forms and Markov Processes, North-Holland, (1980).

[60] M. Gromov, Metric Structures for Riemannian and Non-Riemannian Spaces, Birkhäuser, Boston (1999).

[61] L. V. Kantorovich, "On one effective method of solving certain classes of extremal problems", Dokl. Akad. Nauk USSR 28 (1940), 212-215.

[62] L. V. Kantorovič, G. Š. Rubinšteŭn, "On a functional space and certain extremum problems", (Russian) Dokl. Akad. Nauk SSSR (N.S.) 115 (1957), 1058-1061; "On a space of completely additive functions", (Russian) Vestnik Leningrad. Univ. 13 (1958), 52-59.

[63] N. Ozawa, M. Rieffel, "Hyperbolic group $C^{*}$-algebras and free-product $C^{*}$-algebras as compact quantum metric spaces", Canad. J. Math. 57 (2005), no. 5, 1056-1079.

[64] I. C. Palmer, Noncommutative Geometry and Compact Metric Spaces, PhD Thesis, Georgia Institute of Technology, (May 2010).

[65] B. Pavlović, "Defining metric spaces via operators from unital $C^{*}$-algebras", Pacific J. Math. 186 (1998), 285-313.

[66] M. Rieffel, "Metrics on states from actions of compact groups", Doc. Math. 3 (1998), 215-229.

[67] M. Rieffel, "Metrics on states spaces", Doc. Math. 4 (1999), 559-600.

[68] M. Rieffel, "Compact quantum metrics spaces", in Operator Algebras, Quantization, and Noncommutatve Geometry: a Centennial Celebration Honoring John von Neumann and Marshall H. Stone, (DoRAN, R. S. and Kadison, R. V., eds.), vol. 365 of Contemporary Mathematics, AMS, 2004, pp. 315-330.

Jean Bellissard, Georgia Institute of Technology, School of Mathematics, Atlanta GA $30332-$ 0160

E-mail address: jeanbel@math.gatech.edu 